Hypothesis

\title{
Hypothesis: Induction of biomarkers for detection of colonic neoplasms
}

\author{
Michael Bordonaro, Darina Lazarova ${ }^{凶}$ \\ Department of Basic Sciences, Geisinger Commonwealth School of Medicine, 525 Pine Street, Scranton, PA 18509, USA \\ $\triangle$ Corresponding author: Geisinger Commonwealth School of Medicine, 525 Pine Street, Scranton, PA 18509 Tel: 570-504-9645 Fax: 570-504-9636 Email: \\ dlazarova@som.geisinger.edu \\ (C) Ivyspring International Publisher. This is an open access article distributed under the terms of the Creative Commons Attribution (CC BY-NC) license \\ (https://creativecommons.org/licenses/by-nc/4.0/). See http://ivyspring.com/terms for full terms and conditions.
}

Received: 2017.08.29; Accepted: 2017.10.18; Published: 2018.01.01

\begin{abstract}
The signing of the National Cancer Act of 1971 by President Nixon marked the beginning of our war on cancer. More than 45 years later, the war is still going steady, with the enemy being almost as strong as in 1971. Furthermore, the increasing rates of obesity not only among adults, but among children and adolescents, are the likely cause for the 30-year trend of colon cancer (CC) becoming a disease of the younger population in the U.S. These trends, however, have not spurred the development of novel screening approaches for CC. Considering the need for a sensitive and non-invasive detection of early stage neoplastic lesions in the colon, we propose the development of a test based on a novel concept the concept of induced biomarkers. The proposal is based upon our findings that the food additives propolis and gamma-cyclodextrin (gCD) (a) decrease the neoplastic burden in normal weight and obese ApcMin mice, a model of early stage intestinal neoplasia, and (b) elicit significant changes in the serum proteome in ApcMin mice. We posit that $\mathrm{gCD}$ and propolis induce the release of neoplasm-associated biomarkers in systemic circulation (e.g., metabolites, neoplastic, apoptotic, and immune response proteins), and these markers could be used to detect early stage intestinal neoplasms. Additional dietary bioactives may also elicit a complement of induced markers. The hypothesis could be ascertained by

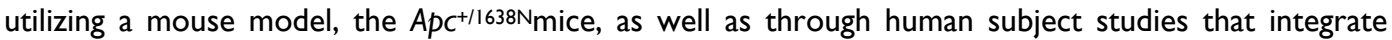
proteomics and metabolomics analyses. The concept of detecting inducible markers of colonic neoplasms is novel, and is substantiated by the significant physiological effects of gCD and propolis on neoplastic colonic cells in culture and on early neoplastic development in ApcMinmice. The long-term objective is to develop a minimally invasive method that detects early stage neoplastic development in the human colon.
\end{abstract}

Key words: colon cancer, inducible markers, metabolites, $A p c^{\text {Min }}$ mice.

\section{Introduction}

Additional research on colorectal (CRC) screening is needed, concluded the 2016 report of USPSTF [1]. The report specified that "...colonoscopy, flexible sigmoidoscopy, computed tomographic colonography, and stool tests have differing levels of evidence to support their use, ability to detect cancer and precursor lesions, and risk of serious adverse events in average-risk adults. Although CRC screening has a large body of supporting evidence, additional research is still needed." A 2016 JAMA editorial also concluded that there is no randomized clinical trial or other high-quality evidence showing that colonoscopy reduces CRC mortality, and the only tests shown to reduce CRC mortality are periodic FOBT and a one-time flexible sigmoidoscopy [2]. As of 2016, the Canadian Task Force on Preventive Health Care recommends CRC screening for adults aged 50 to 74 years with FOBT (gFOBT or FIT) every two years or flexible sigmoidoscopy every 10 years [3]. Current European guidelines also do not recommend colonoscopy screening, unless fecal blood is detected or a sigmoid exam is abnormal.

Despite the fact that the recommended high sensitivity FOBT (guaiac or immunochemical) is a noninvasive and inexpensive approach, the test detects primarily cancer and not adenomatous polyps (i.e., the early stage of neoplastic development). 
Sigmoidoscopy detects neoplasms only in the distal third of the colonic lumen, and it requires bowel preparation. Therefore, the development of new non-invasive methods to detect early stage colonic neoplasms is critically needed.

Here we propose a detection approach of colonic neoplasms that is based upon induced biomarkers. Unlike other neoplastic lesions, colonic neoplasms are in direct contact with dietary agents and their metabolites in the luminal content of the colon. Therefore, dietary agents that affect the physiology of neoplastic colonic cells could be applied as challenges (stimuli) to induce metabolic changes, as well as neoplastic, apoptotic, and immune response markers. For example, many dietary agents induce differential signaling changes in mutant and normal colonic cells, and lead to apoptosis of mutant cells. In vivo, apoptosis is followed by the release of neoantigens from the dying cells. Both driver and passenger mutations in neoplastic cells can give rise to neoantigens, some of which are immunogenic. During an immune response to neoantigens, in addition to the induction of cytokines such as IL-1beta, IL-6, IL-8, TNFalpha [4, 5], the levels of other immune response-associated proteins are altered, and both, positive and negative acute-phase reactants are detected in systemic circulation.

Pro-apoptotic dietary agents may trigger: (a) higher expression and release of neoantigens in systemic circulation, (b) changed expression of apoptotic markers, (c) altered circulation of immune response-associated proteins, and (d) metabolite differences in sera. All of these changes can be registered by comparative analyses of sera before and after administration of dietary supplements, with the objective of detecting colonic neoplasms. Based upon our previous studies [6], we propose that gamma-cyclodextrin (gCD) and propolis induce biomarkers (e.g., neoplastic proteins, apoptotic markers, immune response proteins, metabolites) that are released in the systemic circulation, and the pattern and level of expression of the markers correlate with the presence or absence of intestinal neoplasms.

The hypothesis could be ascertained by utilizing $A p c^{+} / A p c^{1638 N}$ mice. In these mice, neoplastic development in the intestines progresses to carcinoma within the first year of life, and obesity almost doubles the intestinal neoplastic burden. Currently, there is an urgent need to develop non-invasive methods that detect early stage colonic neoplasms in the context of normal weight and obesity. This is dictated by the fact that two out of three CC patients in the U.S. are with above normal body-mass index (BMI). Higher than normal BMI is associated with an increased risk of the predominant form of colorectal cancer (CRC), microsatellite stable (MSS) CRC [7]. In addition, a recent report revealed a trend of CRC becoming a disease of younger individuals. Thus, CRC incidence has increased among 20- to 34-year olds in the past 35 years and, if the trend continues, there will be up to a $90 \%$ increase in colon cancer (CC) incidence in this age group by 2030 [8]. This dramatic shift in age of incidence is likely due to the increasing obesity among children and adolescents [8,9]. Indeed, considering that CC progression takes 5 to 20 years, a simple calculation indicates that CCs diagnosed at age of 20 to 34 might be initiated in school-age children.

The innovation of the proposed approach is conceptual. First, despite the fact that $2 / 3$ of the adult U.S. population is overweight/obese, most CC studies have neglected this fact. Our analyses of The Cancer Genome Atlas (TCGA) data indicate that from all CC patients with reported BMI ( $\mathrm{n}=175$ at the time of the analyses), $33.1 \%$ are with normal weight, $34.9 \%$ are overweight, and $32.0 \%$ are obese [10]. This BMI distribution differs for other types of cancer; for example, the majority of liver cancer patients in TCGA's database are with normal BMI (data not shown). Second, current cancer detection approaches focus on naturally arising neoplastic biomarkers or imaging. Such markers are at low levels, and differ among same-anatomic location neoplasms in different individuals. The idea of detecting neoplasms by detecting induced markers (e.g., immune response-associated proteins and metabolites) has never been tested. Immune-response proteins have already been identified in sera of patients with oral squamous cell carcinoma [11]. C-reactive protein, associated with immune response, has been proposed as a marker for CC risk [12]; detection of antibodies to specific immunogenic tumor proteins has been discussed as a diagnostic method [13-16]. However, these studies have not relied on challenging the neoplasms with the objective of amplifying/inducing a diagnostic signature. The proposed "neoplasm-challenge" approach of detection is unique in its potential to elicit robust physiological changes that are easy to detect. We predict that the neoplasm-challenge approach will result in (a) amplified levels of already identified biomarkers, and (b) identification of novel markers (e.g., neoplasm-associated metabolite and immune-response proteins).

\section{Premise}

Neoplastic and normal colonic cells respond differently to innocuous challenges, such as dietary compounds and their metabolites. One example is butyrate, a metabolite of gamma-cyclodextrin (gCD) 
and dietary fiber. The physiological concentrations of butyrate in the large intestine can reach a maximum of 10-20 mM [17]. Exposure to butyrate concentrations as low as 2 to $5 \mathrm{mM}$ leads to (a) hyperactivation of WNT/beta-catenin signaling in CC cells with mutations-deregulated pathway, and (b) subsequent apoptosis of these cells [18]. Normal colonocytes, however, metabolize butyrate as a source of energy [19], do not hyperactivate WNT/beta-catenin signaling (since they do not have mutations in the pathway), and do not undergo apoptosis in the presence of butyrate. In vitro studies in our laboratory have established that butyrate-induced apoptosis of CC cells is augmented by suppressing AKT and STAT3 survival signaling, as achieved by co-treatment with propolis, a honeybee product available as a dietary supplement $[20,21]$.

Butyrate, a colonic metabolite of gCD [22-24] and fiber, induces cell growth arrest and apoptosis of colonic cells within the entire range of neoplastic progression: from early adenoma to late carcinoma stage. Studies on the effects of fiber, the dietary source of butyrate, have reported an inverse association of fiber intake with adenoma risk [25, 26]. Consistent with these findings, the colonic microadenoma cell line LT97 is highly sensitive to the effects of butyrate and propolis in vitro [20,27, 28]. In the presence of butyrate, human colon adenoma LT97, colon carcinoma HCT-116, SW620, and HCT-R cells upregulate the levels of 180 mRNAs, 80 of which encode secreted proteins, as determined by the review of The Human Protein Atlas. Gene expression in the four neoplastic colonic cell lines was analyzed with Agilent human whole genome oligo microarrays (data not published). The results support our scientific premise that butyrate-induced physiological changes in both adenoma and carcinoma colonic cells are accompanied by increased expression of secreted and apoptotic proteins, some of which could enter the systemic circulation.

Evidence for neoantigen expression in early stage intestinal neoplasms exists. Mutations in APC are gatekeeping mutations in almost $80 \%$ of all sporadic human CC cases; however, there is evidence that additional (mostly passenger) mutations accumulate at even early stages of neoplastic development. We have established that intestinal adenoma development in $A p c^{\text {Min }}$ mice is characterized by multiple, albeit highly variable, types of mutations [6]. We have sequenced the neoplastic exomes of five normal weight and five obese mice. Mouse exome sequencing was performed on genomic DNA extracted from paraffin-embedded small intestines, as the majority of neoplasms in $A p c^{\mathrm{Min}}$ mice arise in this part of the intestines. The detected alterations were categorized as high, moderate, or low impact variants. High impact variants are assumed to have disruptive impact in the protein (e.g., truncations, loss of function, causing nonsense mediated decay); whereas, moderate impact variants are non-disruptive variants that still could change protein effectiveness (e.g., missense variants, in-frame deletions, etc.). We did not include low impact variants in our analyses, since these mutations are unlikely to change protein behavior (e.g., synonymous variants), and therefore, they do not result in neoantigens. In addition to the germline mutation in $A p c$, there were seven other genes with high impact mutations (Bub1b, Fubp1, Hsp90ab1, Trrap, Crtc1, Hoxa13, Rhoa). A total of 80 genes were affected by high or moderate impact mutations, with an average of $26.9 \pm 8.4$ variants per mouse (normal weight mice exhibited on average 30.6 \pm 6.6 variants and obese mice $-23.2 \pm 9.0$ variants). Note that the values do not apply to a single neoplastic lesion, but represent all adenomas in a single mouse intestine. Similarly, exome sequencing of human colonic adenomas has revealed the presence of multiple mutations $(\mathrm{n}=12)$ even at the early stage of neoplastic development [29, 30]. All mutations, including the passenger mutations could give rise to immunogenic neoantigens. Therefore, it is not surprising that the overall mutation loads (that include passenger mutations), rather than any specific driver gene mutation profiles, associate with the immune response in cancer patients [31]. In conclusion, not only carcinomas, but also adenomas are characterized by multiple mutations, some of which may give rise to immunogenic neoantigens.

Our inducible marker concept is also supported by the ability of neoantigens to elicit immune response. A recent study established that even in cancers with a low mutation burden (cancer of the bile duct, esophagus, colon, rectum, and pancreas), 9 out of 10 patients have mutation-reactive $\mathrm{T}$ cells that recognize up to three epitopes derived from somatic mutations in the tumor [32]. We posit that the presence of immunogenic mutants in early colonic neoplasms can be exploited for the development of diagnostic tools (e.g., detection of immune response-associated proteins, but not of neoantigen-specific antibodies). The expression of immunogenic neoantigens can be increased by the administration of "a challenge", such as dietary supplements that are pro-apoptotic in neoplastic cells. Supporting evidence comes from preliminary studies, in which we compared the serum proteomes of normal weight $A p c^{\text {Min }}$ mice fed a control diet, a diet with $6 \% \mathrm{gCD}$, or a diet with $8 \%$ propolis supplement (containing 6\% gCD). The comparative proteomic analyses between mice on a control diet and mice on a 
diet supplemented with propolis/gCD revealed differences in 44 proteins; 24 of which with a potential immune response function exhibited increased levels of expression (1.5 - 3.5 fold) in mice receiving propolis/gCD (data not published). Three of the 44 differentially expressed proteins exhibited decreased levels of expression and they also have a potential immune response function. Comparison between mice on a control diet and mice on a diet supplemented with $6 \% \mathrm{gCD}$ revealed differences in 16 proteins.

Interestingly, propolis (Propolis with Cyclopower, containing $6 \%$ gCD, Manuka Health New Zealand) effectively inhibits the neoplastic burden in $A p c^{\mathrm{Min}}$ mice with normal weight; whereas, gCD alone more effectively counteracts the neoplastic burden in obese $A p c^{\mathrm{Min}}$ mice. Propolis has been a long-standing dietary supplement in many countries in the world; however, gCD is a recent addition to processed foods at levels of $1 \%$ to $20 \%$ (e.g., dry mixes for beverages, soups, dressings, etc.). gCD is also used as a carrier for vitamins and dietary supplements. Alpha-, beta, and gamma-cyclodextrins are cyclic compounds built by six, seven, or eight glucose units respectively. These compounds are hydrolyzed by alpha-amylases; however, salivary and pancreatic alpha-amylases act slowly on cyclodextrins [22]. Therefore, intact cyclodextrins reach the colon, where the resident microbiota metabolizes them to short-chain fatty acids [22]. In rats, intake of dietary cyclodextrins results in more than a two-fold increase in acetate, propionate, and butyrate concentrations in the cecum [33], and in vitro fermentation of cyclodextrins [with fecal matter from dogs] revealed that from the three types of cyclodextrin, gCD results in a maximal rate of short-chain fatty acid production [24].

The effect of gCD on intestinal neoplastic burden in mice [6] could be explained by the metabolism of gCD to butyrate, as this short-chain fatty acid induces apoptosis in neoplastic intestinal cells. This apoptotic activity of gCD would impact both neoplastic initiation (number of lesions) and progression (size of lesions). In agreement with this possibility, we have detected a decrease in the average size and number of lesions in $A p c^{\mathrm{Min}}$ mice exposed to diets containing $\mathrm{gCD}$, and the differences were statistically significant in the context of Western diets [6]. The ability of gCD to induce apoptosis was supported by our findings that the levels of the pro-apoptotic protein Bax and the cleaved Caspase 3 (a hallmark of apoptosis) were increased in intestinal tissues of mice on a Western diet with gCD [6]. Among $A p c^{\text {Min }}$ mice with normal weight, the propolis supplement (containing 6\% gCD) more effectively reduced neoplastic burden [6]. Based upon in vitro studies, this effect is likely due to not only the induction of apoptosis, but also to the ability of propolis to suppress specific proliferative pathways in neoplastic cells (e.g., JAK/STAT, AKT) $[20,21]$.

Finally, our approach is supported by the findings that the metabolic profile of cancer patients changes and, anecdotally or not [34], these changes allow dogs to "sniff cancer". Although research on cancer-specific metabolites is currently ongoing, no one has explored the ability of dietary supplements to elicit the production of neoplastic cell-specific metabolites. This research direction is relevant, since in addition to the direct metabolism of the supplements, the immune response of the organism to neoantigens also triggers metabolic changes. For example, a recent publication reported detectable in vivo metabolomic changes induced by antigen exposure in calves [35].

\section{Testing the hypothesis, expected results, and conclusions}

Based upon these data, we posit that challenging the intestinal neoplasms with pro-apoptotic dietary supplements induces and amplifies a diagnostic signature, and this signature can be detected in serum. The neoplasm-challenge approach of detection has the potential to elicit robust physiological changes that are easily detected. We propose that parallel proteomic and metabolomic analyses of sera samples from $\mathrm{Apc}^{+/ 1638 \mathrm{~N}}$ mice and wild-type mice before and after exposure to a challenge (i.e., dietary supplements) could reveal a neoplasm-specific signature of markers. The proposed experimental approach is depicted in Fig. 1.

The proposed detection of colonic neoplasms has the potential to be successful since: (a) the approach does not rely only on mutations in known driver genes, some of which occur at late stages of neoplastic development and differ between individuals, (b) the approach does not rely on any particular set of mutated genes, as the neoplastic mutation profiles differ between individuals, (c) the approach relies on the amplified physiological response of the immune system to any neoantigens, rather than on detecting antibodies to diverse neoantigens, (d) the approach relies on the detection of a panel of proteins and metabolites associated with physiological changes induced in by "challenged" neoplastic cells (i.e., apoptosis and/or growth arrest at the cell level, and immune response and metabolic changes at the organism level), and such changes are expected to exhibit less inter-individual variations.

Untargeted proteomic and metabolomic analyses will be performed as depicted in Fig. 2 


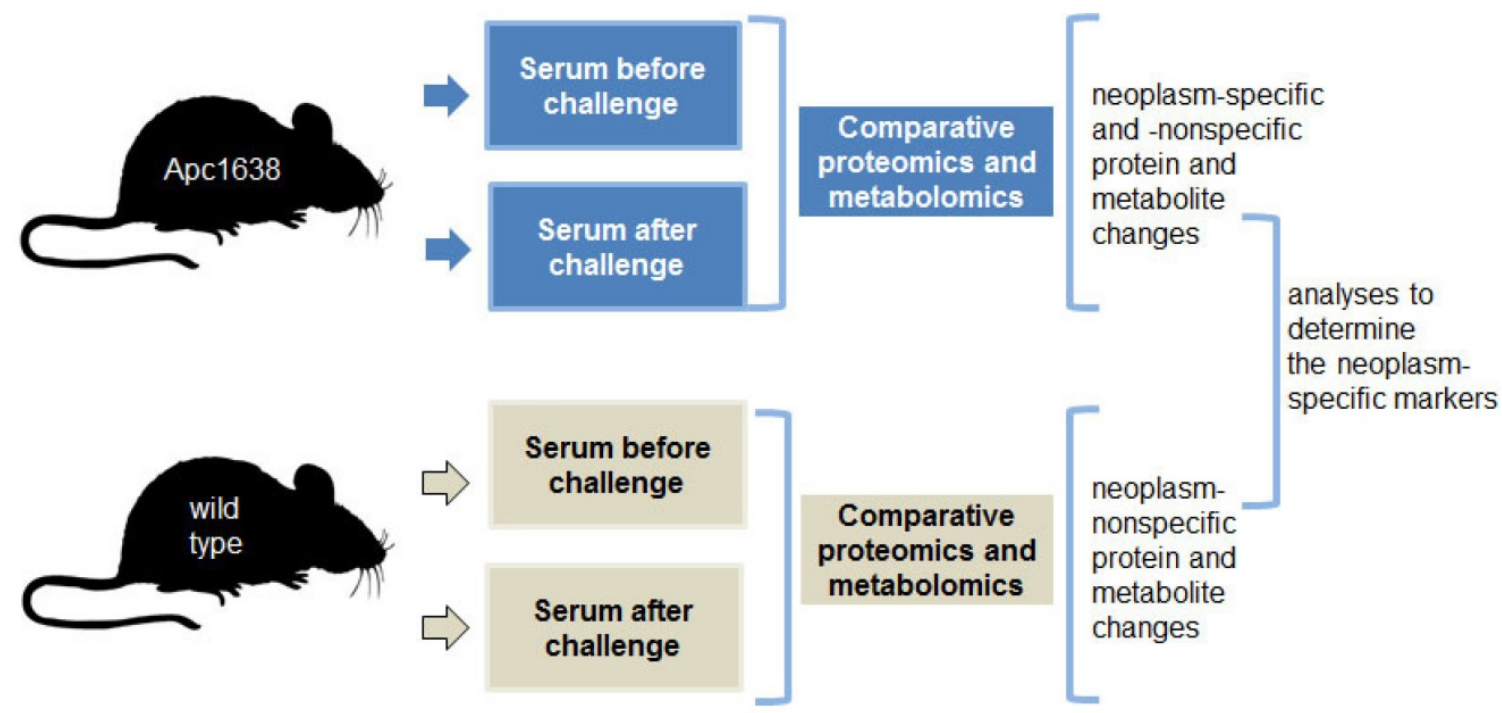

Figure 1. Comparative analyses of sera before and after "a challenge" ( $\mathrm{gCD}$ in obese mice and a propolis supplement in normal weight mice).

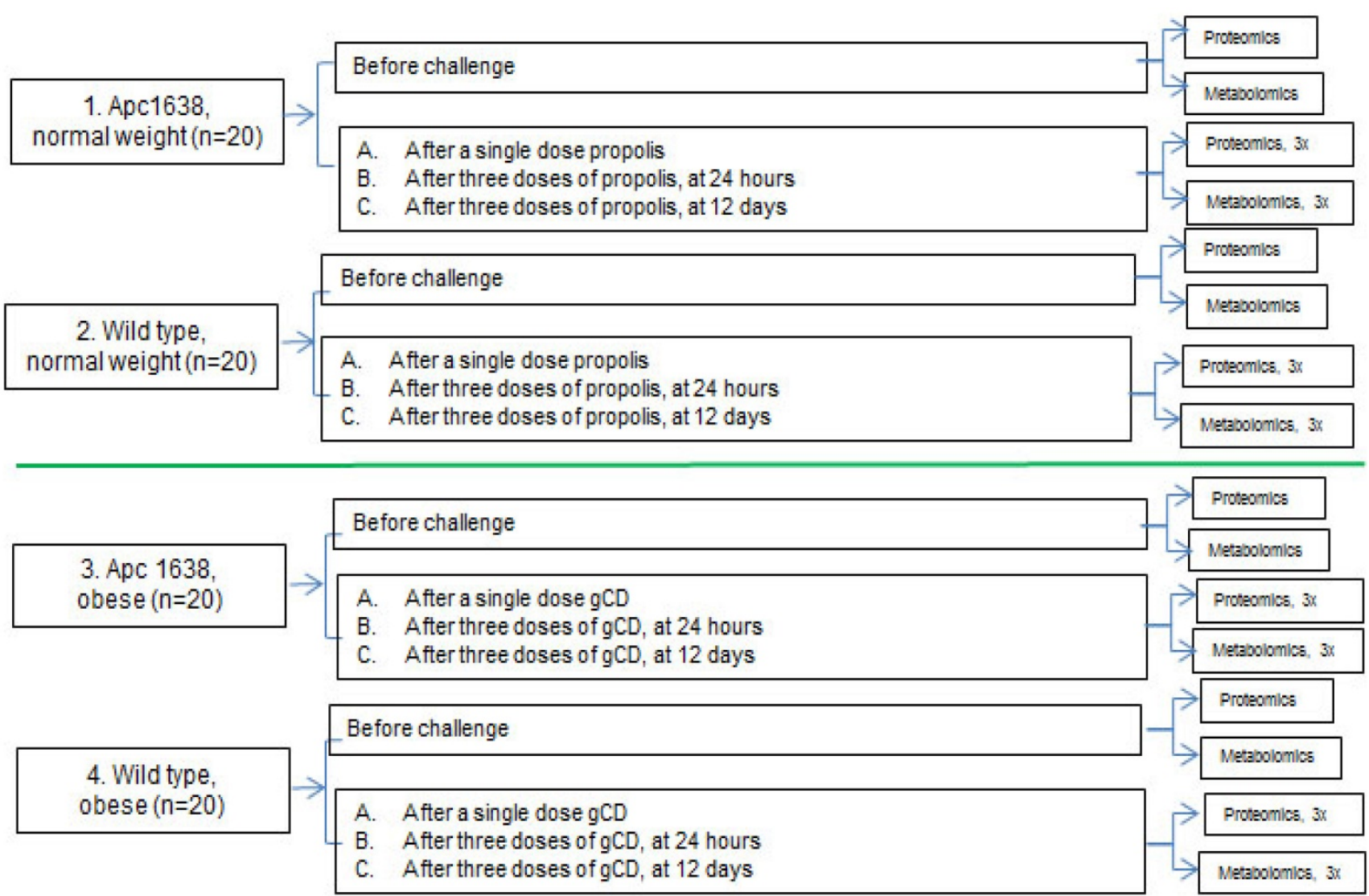

Figure 2. Schematic representation of the blood sampling for parallel proteomics and metobolomics analyses. A total of 32 samples will be analyzed at the proteomics and metabolomics levels. Blood collection and sample analysis will be carried out with mice at age of $5-6$ months, in order to detect early stages of neoplastic development.

The untargeted metabolomic profiling would consist of global analysis of metabolites with molecular weights less than $1 \mathrm{kDa}$, performed, e.g., by Ultra Performance Liquid Chromatography (UPLC) coupled to a highly accurate mass spectrometer that allows for separation and determination of elemental composition (and hence the identification) of thousands of metabolites in a single sample. Prior to metabolic profiling, the metabolites in each sample would be isolated and enriched by a liquid-liquid extraction protocol which enables polar and lipid metabolites to be separated into two fractions. The resultant raw LC-MS data obtained would then be processed to identify discriminating metabolites of 
interest. The proteomic analyses would follow a standard protocol of depleting the sera of abundant proteins such as albumin using ASKs kit, determining the protein concentration using the ToPA Bradford assay kit, reduction and alkylation of the samples, precipitation of the interfering substances, digestion with proteomics grade trypsin and labeling with TMT reagents, followed by Multidimensional Protein Identification Technology via SCX fractionation.

We expect that the challenges (gCD and propolis) will modify/induce a differential spectrum of proteins in sera from $A p c^{+/ 1638 \mathrm{~N}}$ mice and wild type mice. The expectation for the apoptotic release of neoantigens, some of which could be immunogenic, is based upon the knowledge that even early stage neoplastic development in the colon exhibits multiple mutations in addition to the gatekeeping mutation in APC. As neoplastic progression takes place, the number of driver and passenger mutations increases. For example, human colonic adenomas have approximately 12 mutations [29]; whereas, our TCGA analyses revealed that human MSS colon adenocarcinomas in normal weight, overweight and obese patients have on average $147.9 \pm 72.7,123.0 \pm$ 53.9 , and $124.3 \pm 57.7$ somatic mutations, respectively [10]. Although only three to five of these mutations are in driver genes, all mutated proteins have the potential to be immunogenic, and therefore, could be recognized by the immune system upon neoplastic cell apoptosis.

Although the most practical approach to detect inducible biomarkers is to analyze blood samples before and after a challenge, for the detection of colonic neoplasms, stool and urine samples are also potential sources of markers. Another viable approach to detecting neoplasms is that of identifying circulating neoplastic cells. Exposure of neoplastic colonic cells to butyrate decreases their substrate adhesion in vitro [18], and therefore, in vivo exposure to gCD-derived butyrate may result in increased neoplastic cell circulation. Circulating neoplastic cells are a source of identifiable mRNA and DNA. Therefore, future studies to validate our overall hypothesis would focus on analyzing before- and after-challenge RNA and DNA samples for mutations or epigenetic alterations common in colonic neoplasms, and for already established CC markers such as methylated Septin9. The detection of methylated Septin9 has resulted in the development of the Epi proColon test that is currently at the ADMIT stage in the U.S. We expect that the "neoplasm-challenge" approach can greatly increase the reliability of this test or any other proposed/existing test.

Future studies with human subjects should validate promising results from this project. Currently, gCD is generally recognized as safe (GRAS status) by the Food and Drug Administration The average daily intake of gCD from its uses in food has been estimated at $4.1 \mathrm{~g} /$ person / day; whereas, the estimate at the $90^{\text {th }}$ percentile consumption level is 9.0 g / person / day [36]. gCD has been extensively examined for toxicity; its oral, intraperitoneal or subcutaneous administration has not produced any immediate or delayed adverse effects in mice and rats [37]. The tolerance of orally administered gCD has been examined in a double-blind, placebo-controlled, crossover study in 24 healthy volunteers, and a single dose of $8 \mathrm{~g}$ gamma-cyclodextrin was well tolerated [36]. In terms of achievable gCD dosage in humans, the $6 \% \mathrm{gCD}$ intake in mice is equivalent to $3.6 \mathrm{~g}$ of gCD daily intake in humans. This amount was estimated by taking into account the daily food intake for mice ( $\sim 5$ grams of food / day) and the conversion index of $\mathrm{NIH} / \mathrm{NCI}$ [38]. The intake of $3.6 \mathrm{~g} \mathrm{gCD}$ a day is lower than the estimated three-day average daily intake of gCD from 19 foods is at $\sim 4.0 \mathrm{~g}$ [39].

Variation in genetic background, age and lifestyle factors (e.g., physical activity, diet, etc.) would affect the profile of any inducible biomarkers. Therefore, future challenges to serum proteomics and metabolomics, which can be used to identify and evaluate tumor biomarkers, are to differentiate between these inter-individual variations and focus on the neoplasm-induced panel of markers. Proper identification of such markers would require human studies that integrate (a) lifestyle (e.g., diet and activity) considerations, other environmental factors, and background genetic variation; (b) molecular changes in neoplastic tissue (identified at the genetic, epigenetic, proteomic, and metabolomics levels) that can be effectively utilized as biomarkers; and (c) the subset of those biomarkers efficiently induced by agents such as gCD or propolis supplement. Thus, such human subject studies would by necessity overlap with the new interdisciplinary field of molecular pathological epidemiology (MPE) [40, 41]. MPE has particular relevance to disorders such as CRC that have a strong environmental/lifestyle aetiological component [40]. MPE in cancer integrates evaluation of various risk factors, including lifestyle (diet and activity being of paramount important for $\mathrm{CRC}$ ), other environmental factors, and pre-existing genetic variation with molecular classification (e.g., gene mutations, epigenetic changes) of tumors, and then considers how molecular changes interact with the aforementioned risk actors to affect diagnostics, prognosis, and/or potential response to treatment [40]. Of particular relevance to our proposed approach, identifying the relationship between 
particular exposures (i.e., gCD) and the molecular changes characteristic of particular cancers can assist in identifying the appropriate tumor biomarkers for early diagnosis. Case-case studies of human subjects could identify potential biomarkers associated with lifestyle, while case-control studies can identify neoplastic-specific markers [40].

Thus, the MPE approach can be viewed as a supplement, or a complement, to animal studies to assist in the identification of inducible tumor biomarkers. The MPE approach can also extend the concept of inducible tumor biomarkers; thus, findings can be utilized to determine whether particular biomarkers would have greater predictive value when utilized with patients characterized by specific lifestyle and other risk factors; MPE can identify that subset of lifestyle-associated markers induced by gCD or propolis supplement. For example, in the process of colonic carcinogenesis, obesity, smoking, diet and other lifestyle factors affect initiation and progression, and the relevant biomarkers associated with each lifestyle factor and disease risk can be identified [41], allowing for the possibility of inducible biomarker tests tailored for particular subsets of patients (overweight/obese vs. normal weight, smokers vs. non-smokers), which would likely enhance the sensitivity and specificity of the diagnostic tests. MPE is particularly useful in complex hypotheses testing [41], which in the context of our proposed approach would allow for an evaluation of the precise relationship between specific exposures (e.g., to lifestyle factors) and the inducible biomarkers characteristic of different molecular subtypes of neoplasms. In addition, as the inducible biomarker profile is likely to change with disease progression [41], and as lifestyle factors would affect progression, MPE is the most efficient method to approach the problem of identifying the optimal sets of biomarkers for disease stage progression in different cohorts of patients.

Finally, while MPE can obviously be helpful in optimizing diagnostics, conversely, inducible biomarkers identified with MPE, coupled with known lifestyle and other factors, can inform with respect to prognosis and/or response to treatment for diagnosed patients.

Future human subject studies to validate the "neoplasm-challenge" detection approach could involve individuals scheduled for colonoscopy (Fig.3). In these studies prior to colonoscopy, before- and after-challenge blood samples will be collected. The differential levels of the candidate biomarkers will be correlated to positive and negative colonoscopy findings.

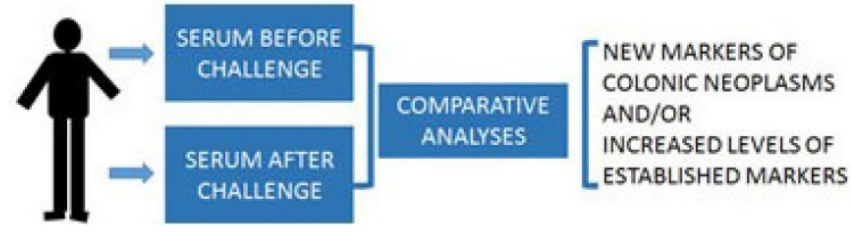

Figure 3. Individuals scheduled for colonoscopy will be consented for blood samples before and after a challenge (e.g., an oral dose of gCD or a propolis supplement). Comparative proteomic and metabolic analyses will be performed. Differential markers will be correlated to colonoscopy results.

In conclusion, the "inducible marker" approach has promise as an optimized non-invasive CC diagnostic toolkit that can increase the level of CC screening. Considering the rapidly increasing pool of younger individuals at risk for $\mathrm{CC}$, non-invasive approaches such as the one proposed by us, scalable for large patient populations, should be a focus of ongoing investigation.

\section{Abbreviations}

CRC: colorectal cancer, CC: colon cancer; gCD: gamma-cyclodextrin

\section{Acknowledgements}

$\mathrm{MB}$ and DL are supported by funding from Geisinger Commonwealth School of Medicine.

\section{Competing Interests}

The authors have declared that no competing interest exists.

\section{References}

1. Lin JS, Piper MA, Perdue LA, et al. Screening for Colorectal Cancer Updated Evidence Report and Systematic Review for the US Preventive Services Task Force. JAMA. 2016; 315: 2576-94.

2. Redberg RF. Fecal blood testing or colonoscopy what is the best method for colorectal cancer screening? JAMA Intern Med. 2016; 176:1071-3.

3. Canadian Task Force on Preventive Health Care. Recommendations on screening for colorectal cancer in primary care. CMAJ 2016, DOI:10.1503/cmaj.151125.

4. Simard JC, Cesaro A, Chapeton-Montes J, et al. S100A8 and S100A9 induce cytokine expression and regulate the NLRP3 inflammasome via ROS-dependent activation of NF-KB. PLoS One 2013; 8:e72138.

5. Idris A, Ghazali NB, Koh D. Interleukin $1 \beta$-A Potential Salivary Biomarker for Cancer Progression? Biomark Cancer. 2015; 7:25-9.

6. Cho Y, Gutierrez L, Bordonaro M, et al. Effects of propolis and gamma-cyclodextrin on intestinal neoplasia in normal weight and obese mice. Cancer Med. 2016; 5: 2448-58.

7. Campbell PT, Jacobs ET, Ulrich CM, et al. Case-control study of overweight, obesity, and colorectal cancer risk, overall and by tumor microsatellite instability status. J Natl Cancer Inst 2010; 102: 391-400.

8. Bailey CE, Hu CY, You YN, et al. Increasing Disparities in the Age-Related Incidences of Colon and Rectal Cancers in the United States, 1975-2010. JAMA Surg 2015; 150: 17-22.

9. Ogden CL, Carroll MD, Kit BK, et al. Prevalence of childhood and adult obesity in the United States, 2011-2012. JAMA 2014; 311: 806-14.

10. Bordonaro M, Lazarova D. Hypothesis: Obesity Is Associated with a Lower Mutation Threshold in Colon Cancer. J Cancer 2015; 6: 825-31.

11. Chen Y, Azman SN, Kerishnan JP, et al. Identification of host-immune response protein candidates in the sera of human oral squamous cell carcinoma patients. PLoS One 2014; 9:e109012.

12. Wu J, Cai Q, Li H, et al. Circulating C-reactive protein and colorectal cancer risk: a report from the Shanghai Men's Health Study. Carcinogenesis 2013; 34:2799-803.

13. Mao J, Ladd J, Gad E, et al. Mining the pre-diagnostic antibody repertoire of TgMMTV-neu mice to identify autoantibodies useful for the early detection of human breast cancer. J Transl. Med. 2014; 12:121. 
14. Katz-Brull R, Seger D, Rivenson-Segal D, et al. Metabolic markers of breast cancer: enhanced choline metabolism and reduced choline-ether-phospholipid synthesis. Cancer Res. 2002; 62:1966-70.

15. Xiao JF, Varghese RS, Zhou B, et al. LC-MS based serum metabolomics for identification of hepatocellular carcinoma biomarkers in Egyptian cohort. J Proteome Res. 2012; 11:5914-23.

16. Zhang $\mathrm{T}, \mathrm{Wu} \mathrm{X}, \mathrm{Ke} \mathrm{C}$, et al. Identification of potential biomarkers for ovarian cancer by urinary metabolomic profiling. J Proteome Res. 2013; 12:505-12.

17. Cummings JH, Pomare EW, Branch WJ, et al. Short chain fatty acids in human large intestine, portal, hepatic, and venous blood. Gut 1987; 28: 1221-7.

18. Lazarova DL, Bordonaro M, Carbone R, et al. Linear relationship between Wnt activity levels and apoptosis in colorectal carcinoma cells exposed to butyrate. Int. J. Cancer 2004; 110: 523-31.

19. Roediger W.E.W. Utilization of nutrients by isolated epithelial cells of the rat colon. Gastroenterology 1982; 83: 424-9.

20. Bordonaro M, Drago E, Atamna W, et al. Comprehensive suppression of all apoptosis-induced proliferation pathways as a proposed approach to colorectal cancer prevention and therapy. PLoS One 2014; 9:e115068.

21. Drago E, Bordonaro M, Lee S, et al. Propolis Augments Apoptosis Induced by Butyrate via Targeting Cell Survival Pathways. PLoS One. 2013; 8:e73151.

22. Szejtli J. Chemistry, physical and biological properties of cyclodextrins. In: Comprehensive Supramolecular Chemistry. Szejtli J, Osa T, eds. volume 3. Pergamon Press, New York; 1996: 5-40.

23. Kaewprasert S, Okada M, Aoyama Y. Nutritional effects of cyclodextrins on liver and serum lipids and cecal organic acids in rats. J Nutr Sci Vitaminol. 2001; 47: 335-9.

24. [Internet] Zubillaga, MAG, Nutritional evaluation of a-cyclodextrin using canine, hamster, and in vitro model, available at http://hdl.handle.net/2142/29583, accessed on November 20, 2015.

25. Platz EA, Giovannucci E, Romm EB, et al. Dietary fiber and distal colorectal adenoma in men. Cancer Epidemiology, Biomarkers \& Prevention 1997; 6: $661-70$.

26. Peters $\mathrm{U}$, Sinha $\mathrm{R}$, Chaterjee $\mathrm{N}$, et al. Dietary fibre and colorectal adenoma in a colorectal cancer early detection programme. Lancet 2003; 361:1491-5.

27. Kautenberger T, Beyer-Sehlmeyer G, Festag G., et al. The gut fermentation product butyrate, a chemopreventive agent, suppresses glutathione S-transferase theta (hGSTT1) and cell growth more in human colon adenoma cells (LT97) than tumor (HT29) cells. J. Cancer Res. Clin. Oncol. 2005; 131: 692-700.

28. Lazarova D, Lee A, Wong T, et al. Modulation of Wnt activity and cell physiology by butyrate in LT97 microadenoma cells. J Cancer 2014; 5: 203-13.

29. Zhou D, Yang L, Zheng L, et al. Exome capture sequencing of adenoma reveals genetic alterations in multiple cellular pathways at the early stage of colorectal tumorigenesis. PLoS One. 2013; 8:e53310.

30. Nikolaev SI, Sotiriou SK, Pateras IS, et al. A single-nucleotide substitution mutator phenotype revealed by exome sequencing of human colon adenomas. Cancer Res. 2012; 72: 6279-89.

31. Van Allen EM, Miao D, Schilling B, et al. Genomic correlates of response to CTLA-4 blockade in metastatic melanoma. Science 2015; 350: 207-11.

32. Tran E, Ahmadzadeh M, Lu YC, et al. Immunogenicity of somatic mutations in human gastrointestinal cancers. Science. 2015; 350: 1387-90.

33. Kaewprasert S, Okada M, Aoyama Y. Nutritional effects of cyclodextrins on liver and serum lipids and cecal organic acids in rats. J Nutr Sci Vitaminol. 2001; 47: 335-9

34. Willis $\mathrm{CM}$, Britton LE, Harris $\mathrm{R}$, et al. Volatile organic compounds as biomarkers of bladder cancer: Sensitivity and specificity using trained sniffer dogs. Cancer Biomark. 2010; 8:145-53.

35. Gray DW, Welsh MD, Doherty S, et al. Identification of systemic immune response markers through metabolomic profiling of plasma from calves given an intra-nasally delivered respiratory vaccine. Vet Res. 2015; 46:7.

36. Munro IC, Newberne PM, Young VR, et al. Safety assessment of gamma-cyclodextrin. Regul Toxicol Pharmacol. 2004; 39 Suppl 1:S3-13.

37. Matsuda K, Mera Y, Segawa Y, et al. Acute toxicity study of c-cyclodextrin (c-CD) in mice and rats. Ogo Yakuri (Pharmacometrics). 1999; 26: 287-91.

38. [Internet] Equivalent Surface Area Dosage Conversion Factors, by NIH/NCI. https://ncifrederick.cancer.gov/lasp/acuc/frederick/Media/Documents/A CUC42.pdf.

39. [Internet] WHO Food Additives Series 44, gamma-Cyclodextrin, International program on chemical safety, World Health Organization. http://www.inchem.org/documents/jecfa/jecmono/v44jec05.htm

40. Ogino S, Chan AT, Fuchs CS, et al. Molecular pathological epidemiology of colorectal neoplasia: an emerging transdisciplinary and interdisciplinary field. Gut. 2011; 60:397-411.

41. Ogino S, Nishihara R, VanderWeele TJ, et al. Review Article: The Role of Molecular Pathological Epidemiology in the Study of Neoplastic and Non-neoplastic Diseases in the Era of Precision Medicine. Epidemiology. 2016; 27:602-11. 